\title{
Reconfiguration of respiratory-related population activity in a rostrally tilted transversal slice preparation following blockade of inhibitory neurotransmission in neonatal rats
}

\author{
Frank Funke • Michael Müller • Mathias Dutschmann
}

Received: 28 December 2007 / Revised: 10 March 2008 /Accepted: 23 March 2008 / Published online: 6 May 2008

(C) The Author(s) 2008

\begin{abstract}
Recent studies showed that respiratory rhythm generation depends on oscillators located in the pre-Bötzinger complex (pre-BötC) and the parafacial respiratory group (pFRG). To study inhibitory synaptic interactions between these two oscillators, we developed a rostrally tilted transversal slice preparation, which preserves these regions. The onset of rhythmic mass activity in the retrotrapezoid nucleus (RTN)/ pFRG preceded that of the pre-BötC. Blockade of glycinergic and gamma-aminobutyric acidic inhibition synchronized preBötC and RTN/pFRG activity and significantly increased preBötC burst frequency, amplitude, and duration. Population imaging revealed recruitment of inspiratory-like neurones, while expiratory-like neurones lost their phasic activity. The reconfiguration after disinhibition reveals: (1) synaptic inhibition of the pre-BötC arising from the RTN/pFRG, (2) excitatory drive from the RTN/pFRG that triggers the pre-
\end{abstract}

\section{F. Funke $\cdot$ M. Müller $\cdot$ M. Dutschmann}

DFG Research Center Molecular Physiology of the Brain (CMPB),

Zentrum für Physiologie und Pathophysiologie,

Abteilung Neuro- und Sinnesphysiologie,

Georg-August-Universität Göttingen,

Humboldtallee 23,

37073 Göttingen, Germany

F. Funke

e-mail: ffunke1@gwdg.de

M. Müller

e-mail: mmuelle@gwdg.de

\section{Dutschmann ( $\square)$}

Zentrum Physiologie und Pathophysiologie,

Universität Göttingen,

Humboldtallee 23,

37073 Göttingen, Germany

e-mail: mdutsch@gwdg.de
BötC burst. Our findings support the view that these synaptic interactions in vitro relate to the initiation of the inspiratory phase or to the steering of the expiratory-inspiratory phase transition in vivo.

Keywords Rhythmogenesis $\cdot$ Respiration $\cdot$ Respiratory rhythm $\cdot$ Neural network $\cdot$ Calcium imaging

\section{Introduction}

Numerous studies and reviews have addressed the question of how the mammalian respiratory rhythm and motor pattern is generated, modulated, and transmitted to the respiratory muscles [1-16]. Despite the growing knowledge in this field the models for respiratory rhythm generation are still being discussed controversially [17-19].

The respiratory network undergoes maturational changes during the postnatal period. One hypothesis suggests that in particular synaptic inhibition becomes more important for respiratory rhythm generation during ontogeny $[9,10,20,21]$. Nevertheless, synaptic inhibition is required from birth on to generate a physiological threephase motor pattern [22]. The generation of this threephase motor pattern of breathing (inspiration, postinspiration and late expiration) requires the functional and structural integrity of a pontomedullary respiratory network $[3,4$, $14,15,23,24]$. Nevertheless, the network can be separated anatomically and functionally into compartments. Apparently, distinct compartments serve different functions in the generation and modulation of the respiratory rhythm and motor pattern. Pontine areas such as the Kölliker-Fuse (KF), intertrigeminal, and A5 region have strong modulatory influences on the respiratory motor pattern and contribute essentially to the generation 
of the eupnoeic motor pattern, while the primary rhythmgenerating circuits are located in the medulla oblongata. In particular, the medullary regions of the respiratory network such as the pre-Bötzinger complex (pre-BötC) can be separated from the network and maintain their rhythmic activity under in vitro conditions. Consequently, the preBötC was considered the rhythmogenic kernel of the network which initiates the respiratory rhythm $[2,25,26]$. Over the years, this view was further developed [13]. A pioneer study of Onimaru and Homma [27] in 2003 demonstrated, in the en bloc brainstem-spinal cord preparation of newborn rats for the first time, that rhythmic activity of the parafacial respiratory group ( $\mathrm{pFRG}$, in adult rats this region corresponds to the retrotrapezoid nucleus, RTN) might trigger the rhythm of the pre-BötC. This initial finding gave rise to the current hypothesis that the respiratory rhythm is generated by oscillations between two rhythmogenic kernels involving the pre-BötC and the RTN/pFRG [2830]. Thereby, the pre-BötC produces the inspiratory phase because its activity correlates either with the inspiratory hypoglossal [26] or phrenic nerve motor output [31, 32], whereas the RTN/pFRG generates predominantly preinspiratory [13, 27] or expiratory [29] bursts. Because neuronal oscillations classically require synaptic inhibition, glycinergic and/or gamma-aminobutyric acid (GABA)ergic connections could be involved in the coupling of the rhythmgenerating circuits between pre-BötC and RTN/pFRG.

To address the relevance of synaptic inhibition for the generation of in vitro respiratory activities, we developed a rostrally tilted transversal slice preparation of neonatal rat brainstem containing the pre-BötC, the RTN/pFRG, and the pontine regions of the respiratory network. Compared to the tilted sagittal slice preparation which was previously used to study rhythmogenesis [20, 33], the novel slice preparation introduced in the present study preserves the bilaterally distributed respiratory network in both hemispheres and its interconnectivity. Recent advances in technology made it possible to image population activity at single-cell resolution while simultaneously recording rhythmic mass activity $[33,34]$. In this study, we show that distinct rhythms are recordable in the rostrally tilted slice preparation. The rhythmic activity arising from the RTN/ pFRG preceded the rhythm of the pre-BötC. Blockade of inhibitory neurotransmission after bath application of strychnine and bicuculline resulted in an increased burst frequency, amplitude, and duration of the integrated preBötC mass activity. The lengthening of the pre-BötC burst was based on a synchronization of the pre-BötC rhythm to the preinspiratory activity of the RTN/pFRG. Our imaging approach in the tilted transversal slice preparation revealed putative underlying mechanisms on the cellular level. Recruitment of additional inspiratory neurones and a loss of respiratory-correlated activity in expiratory neurones were observed.

\section{Materials and methods}

\section{Preparation}

Rhythmically active slices were prepared from neonatal Sprague-Dawley rats (postnatal day 5-8, either sex). Rats were deeply anesthetized with isoflurane, decapitated, and the brain was rapidly removed and transferred to ice-cold artificial cerebrospinal fluid (ACSF) with a lowered calcium concentration $(0.75 \mathrm{mM})$. The pia mater was removed; the isolated brainstem was glued on an agarose block, and a transverse $700-\mu \mathrm{m}$ slice containing the preBötC was cut with a vibroslicer (752 M, Campden Instruments) at an angle of $47^{\circ}$. To cut the novel slice preparation that contains the pre-BötC, the $\mathrm{RTN} / \mathrm{pFRG}$, and more rostral parts of the respiratory network including pontine areas (Fig. 1), we used the area postrema and the caudal border of the inferior colliculus as main landmarks. The initial cut was performed at two thirds of the distance between area postrema and inferior colliculus in order to expose the pre-BötC. The second cut was performed $700 \mu \mathrm{m}$ rostral in order to maintain brainstem areas rostral to the pre-BötC. The slice was transferred to $\operatorname{ACSF}\left(30^{\circ} \mathrm{C}\right.$, $95 \% \mathrm{O}_{2} / 5 \% \mathrm{CO}_{2}$ ) and was left undisturbed for at least $30 \mathrm{~min}$. Before dye loading, the calcium concentration was adjusted to $1.5 \mathrm{mM}$ (ACSF) and the slice was undisturbed for another $30 \mathrm{~min}$.

At the end of the experiments, the slices were fixed in $4 \%$ paraformaldehyde. For anatomical verification of respiratory-related brainstem nuclei, we cut the slices into $50-\mu \mathrm{m}$ sections using a freezing microtome (Reichert \& Jung, Wetzlar, Germany). Later, the sections were stained with thionin (see Fig. 1).

\section{Solutions}

Unless mentioned otherwise, all chemicals were obtained from Sigma-Aldrich. Standard ACSF was composed of $128 \mathrm{mM} \mathrm{NaCl}, 3 \mathrm{mM} \mathrm{KCl}, 1.5 \mathrm{mM} \mathrm{CaCl}_{2}, 1 \mathrm{mM}$ $\mathrm{MgSO}_{4}, 0.5 \mathrm{mM} \mathrm{NaH}_{2} \mathrm{PO}_{4}, 21 \mathrm{mM} \mathrm{NaHCO}$, and $30 \mathrm{mM}$ dextrose. The ACSF was constantly aerated with carbogen $\left(95 \% \mathrm{O}_{2} / 5 \% \mathrm{CO}_{2}\right)$ to adjust the $\mathrm{pH}$ to 7.4 . Fluo-3 $\mathrm{AM}$ (Mobitec) was dissolved as a $2-\mathrm{mM}$ stock in dimethyl sulfoxide containing $10 \%$ pluronic acid. The dye stock was kept frozen. Bulk loading of slices $\left(45 \mathrm{~min}\right.$ at $30^{\circ} \mathrm{C}$ in $3 \mu \mathrm{M}$ fluo-3 AM) was performed in a custom-built chamber $(3 \mathrm{ml})$ with constant oxygenation. The slices 

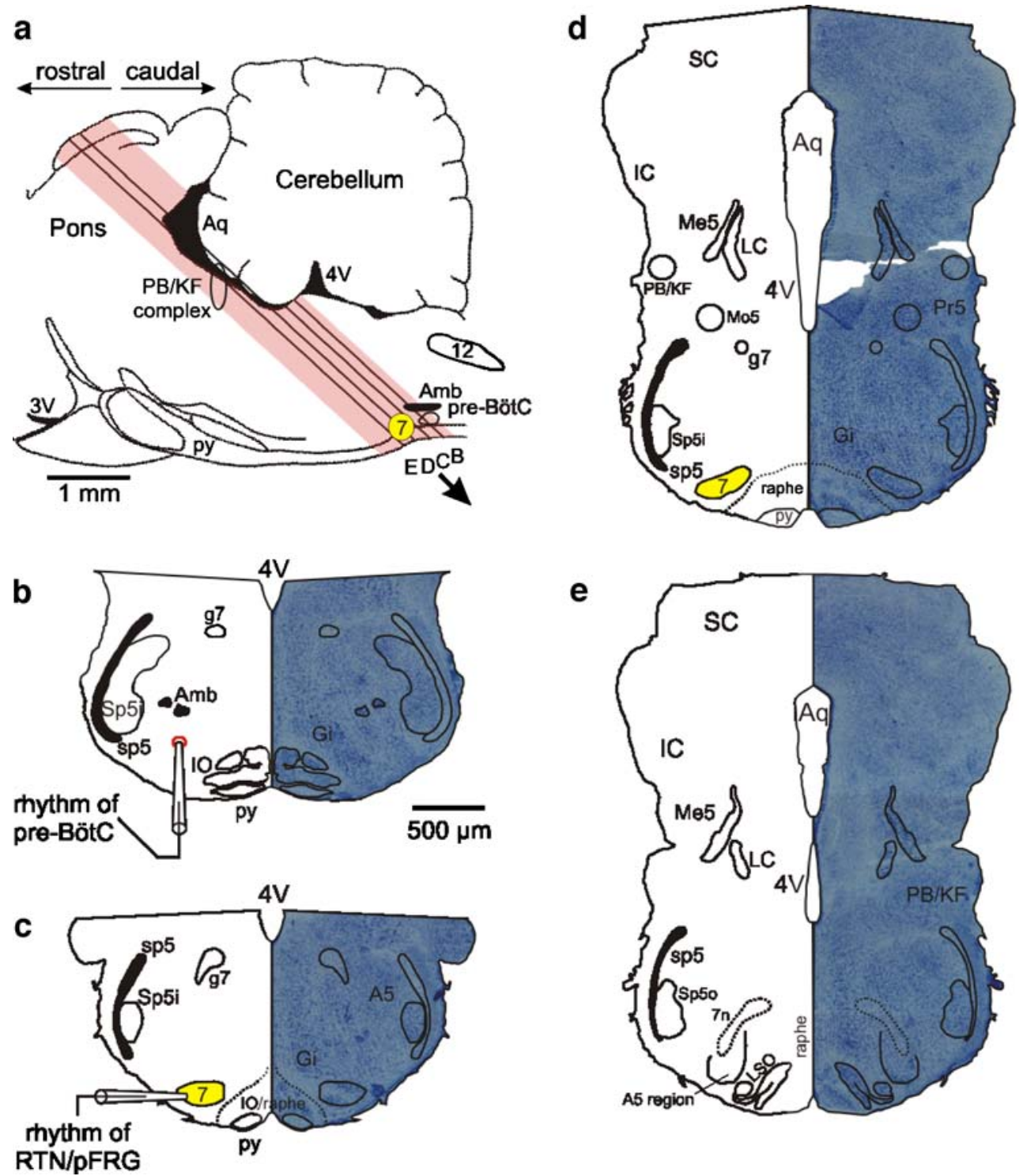

Fig. 1 Anatomical description of the tilted transversal slice preparation. a Schematic drawing of a midline sagittal section through the pontomedullary brainstem showing the $47^{\circ}$ cutting angle for the tilted slice preparation. Note that the laterally located respiratory centers are projected onto this mediolateral section to allow for better comparison with the transversal sections below. Due to this projection, the region of the parabrachial/Kölliker-Fuse complex $(P B / K F)$ misleadingly overlaps with the fourth ventricle. To allow for comparison with the transversal sections shown in $\mathbf{b}-\mathbf{e}$, black lines highlight the location within the $700-\mu \mathrm{m}$-thick slice. The transversal sections (b-e) show the original thionin staining on the right side and a semi-schematic drawing on the left. b This section displays the caudal part of the slice containing the nucleus ambiguus $(A m b)$ and the inferior olive $(I O)$ as two crucial landmarks for the identification of the pre-Bötzinger complex (pre-BötC). The location of the recording electrode for the pre-BötC mass activity is indicated. c The anatomy of the slice in a depth of $200 \mu \mathrm{m}$ from the caudal surface. First facial motoneurones (7)

are detectable on the ventral side while the genu of the facial nerve $(g 7)$ and the noradrenergic A5 region (A5) are visible more dorsally. At this depth, the tip of the second electrode was placed to record RTN/ pFRG mass activity. d This section contains medullary and rostral pontine areas. On the ventral side, the facial motoneurones are present. Crucial dorsal landmarks for the neonatal $(P B / K F)$ such as the principal nucleus of trigeminal tract $(\operatorname{Pr} 5)$, the trigeminal motor nucleus (Mo5), the mesencephalic nucleus of the trigeminus (Me5), and the locus coeruleus $(L C)$ were identifiable [57]. e In the most rostral section, the facial motoneurones fade and the lateral superior olive $(L S O)$, the facial nerve $(7 n)$ and the A5 region become visible ventrally. Dorsally, the rostral pons including the $(P B / K F)$ is still detectable. Abbreviations: $3 \mathrm{~V}, 4 \mathrm{~V}$ third and fourth ventricle; 12 hypoglossal nucleus; $A q$ aqueduct; $G i$ gigantocellular reticular nucleus; $I C$ inferior colliculus; $p y$ pyramidal tract; $S C$ superior colliculus; $s p 5$ spinal trigeminal tract; $S p 5 i$ spinal trigeminal nucleus (interpolar part); Sp5o spinal trigeminal nucleus (oral part) 
rested on a nylon mesh to optimize the circulation and tissue penetration of the dye. One hundred millimolar mannitol was added to the staining solution to widen interstitial space and to improve dye penetration into the tissue [34]. Strychnine and bicuculline were kept as frozen stock solutions of $120 \mu \mathrm{M}$ and $1 \mathrm{mM}$, respectively. The final solutions (strychnine $500 \mathrm{nM}$; bicuculline $20 \mu \mathrm{M}$ in ACSF) were prepared freshly from the frozen stocks shortly before experiment.

\section{Electrical recordings}

Electrical mass activity was recorded extracellularly from the regions of the pre-BötC and RTN/pFRG with singlebarreled microelectrodes pulled from thin-walled borosilicate glass (diameter $1.5 \mathrm{mM}$, Modulohm, Denmark) using a horizontal puller (BMZ Universal Puller, Zeitz Instruments, München, Germany). The tips of the electrodes were broken to a final resistance of $\sim 1 \mathrm{M} \Omega$ and filled with ACSF. Field potentials were amplified by a custom-built amplifier, band-pass filtered (cutoff frequencies: high-pass $400 \mathrm{~Hz}$, low-pass $1.7 \mathrm{kHz}$ ), and digitized at $10 \mathrm{kHz}$ using a PowerLab/4SP interface and Chart 5 software (ADInstruments, Spechbach, Germany). The raw signals were rectified and integrated online $(\tau=200 \mathrm{~ms})$. Further off-line analysis was performed using clampfit 9.2 (Molecular Devices, Union City, USA) and Excel (Microsoft Office 2003).

For recordings of the pre-BötC mass activity, the electrode was placed on the slice surface at an acute angle coming from the ventral side. Rhythmic activity was reliably found ventrally of the nucleus ambiguus, halfway between the ventralmost part of the spinal trigeminal tract and the midline of the slice (Fig. 1b). The (RTN/pFRG) mass activity was recorded at a depth of 100-200 $\mu \mathrm{m}$ via an electrode inserted from the lateral side (Fig. 1c). After placement of the electrodes, baseline activity was recorded for 5 to $10 \mathrm{~min}$ before any drug treatment or stimulation was performed.

\section{Electrical stimulation}

For electrical stimulation (S48, Grass, USA) experiments, Teflon-coated steel-wire electrodes (diameter $=50 \mu \mathrm{m}$, AM-systems) were used. The electrodes were placed in the parabrachial/Kölliker-Fuse complex (PB/KF). Single stimuli or trains $(0.1-2.0 \mathrm{~Hz})$ of various strength $(0.1-$ $1.5 \mathrm{~mA}$ ) and duration $(0.1-0.2 \mathrm{~ms})$ were applied. To avoid stimulation artefacts, a custom-built disconnector was used, blocking the input of the rectifier-integrator during stimulation.
Optical recordings

Optical recordings were performed with a computercontrolled fluorescence imaging system composed of a monochromatic xenon light source (Cairn Optoscan Monochromator, Cairn Research Ltd., Kent, UK) and a highly sensitive charge-coupled device camera (Retiga EXi Fast1394, QImaging, Burnaby, Canada) attached to an upright microscope (BX51, Olympus, Hamburg, Germany). A $20 \times, 0.95$ NA water immersion objective (Olympus XLUMPlanFI) was used.

The dye-loaded slices were placed in a submersion-style chamber at $27^{\circ} \mathrm{C}$ (flow rate $5-6 \mathrm{ml} / \mathrm{min}$ ). Fluo-3 was excited at $490 \mathrm{~nm}$ and fluorescence was recorded using a 515-nm beam-splitter and a 535/50 band-pass filter. Images were taken at a frame rate of $2 \mathrm{~Hz}$ and an exposure time of $50 \mathrm{~ms}$. Two-by-two binning was applied.

Typically, a time series of 500 frames was taken $5 \mathrm{~min}$ after the electrodes were placed and stable rhythms were recorded. Ten minutes after the application of strychnine and bicuculline, two consecutive time series of 500 frames were recorded.

\section{Off-line image processing}

Off-line averaging markedly reduced noise levels. Thus, neurones could be visualized in deeper tissue layers and correlated with pre-BötC mass activity. All images of a given series (time-lapse recording) were corrected for photo bleaching by equalizing background intensity using the photo bleaching compensation algorithm included in the AndorIQ 6.03 image analysis software (Andor Bioimaging, Nottingham, UK) or a Matlab (Matlab 7.1, Mathworks, USA) algorithm developed by us. To enhance changes in pixel brightness and image contrast, the minimum intensity projection of a given image series (i.e., the image composed of the lowest pixel intensities obtained over a whole timelapse recording) was subtracted from each single image. The optically recorded neuronal activity was averaged over several consecutive respiratory bursts. As onset of a respiratory burst, we defined a threshold of $50 \%$ of the burst amplitude (rectified, integrated mass activity). Based on this threshold, nine consecutive images were identified: the image taken at the pre-BötC burst threshold as well as the four preceding and the four following frames. The corresponding images of these nine picture sequences (i.e., the images taken at the same relative time during each preBötC burst) were averaged over 20-40 consecutive pre-BötC bursts [34].

For better illustration of optically recorded cell activities, the picture sequences were color coded with respect to the maximum-minimum of each pixel and its time of occur- 
rence in the sequence using a Matlab algorithm (Matlab 7.1, Mathworks, USA) developed by us. Inspiratory-like activity is displayed in red, expiratory-like in blue. To abolish optical noise, only changes in pixel brightness that exceeded twice the standard deviation of the baseline activity were used.
Statistics

All numerical values are represented as mean \pm standard deviation. Significance of the observed changes was tested using the Student's $t$-test (paired, two tailed). In case of multiple tests, the Bonferroni correction was applied. Signif-
Fig. 2 Dual recording of rhythmic mass activities from the preBötC and RTN/pFRG before and after blockade of inhibitory neurotransmission. a Sample recording of the rectified and integrated mass activity arising from the pre-BötC (upper trace) and $\mathrm{RTN} / \mathrm{pFRG}$ (lower trace).

To illustrate that the RTN/pFRG rhythm preceded that of the preBötC, the preinspiratory activity is highlighted in grey. b After application of $500 \mathrm{nM}$ strychnine and $20 \mu \mathrm{M}$ bicuculline, the rhythms of the pre-BötC and RTN/pFRG became synchronized. The vertical lines mark the onset of both bursts. Please note the increased burst frequency after blockade of inhibitory neurotransmission and the increased amplitude of the rectified and integrated mass activity of the pre-BötC. $\mathbf{c}$ shows the synchronization of the pre-BötC and RTN/pFRG rhythm. The error bars represent the standard deviation. Note that before strychnine-bicuculline application the rising slopes of the rectified and integrated signals of the two rhythms differed significantly, indicating an earlier onset of the RTN/pFRG rhythm compared to that of the pre-BötC. After blockade of inhibitory neurotransmission, the two rhythms became synchronized due to a broadening of pre-BötC bursts. $\mathbf{d}$, e Changes of the instantaneous pre-BötC burst frequency and burst amplitude after blockade of inhibitory neurotransmission are illustrated. The averages of 19 slices are plotted
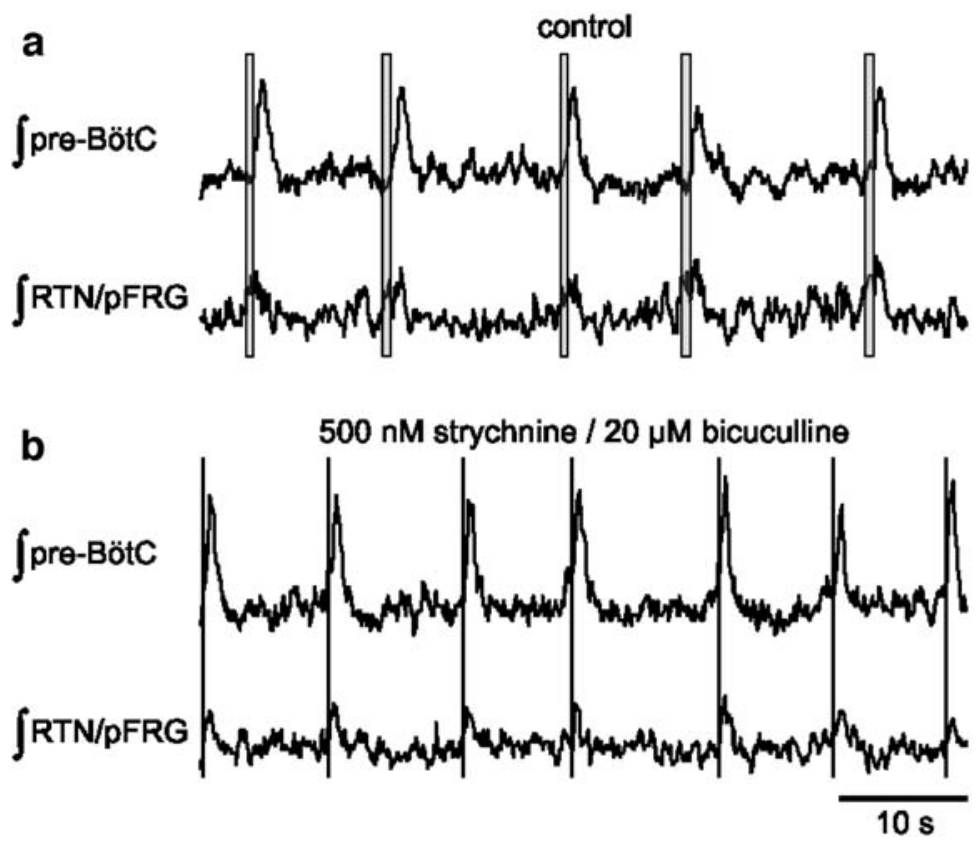

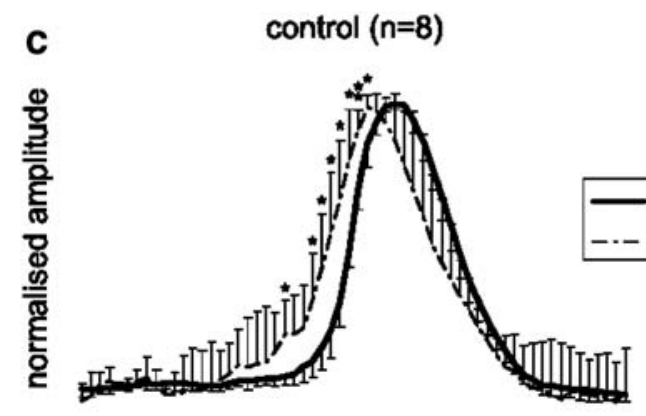

d

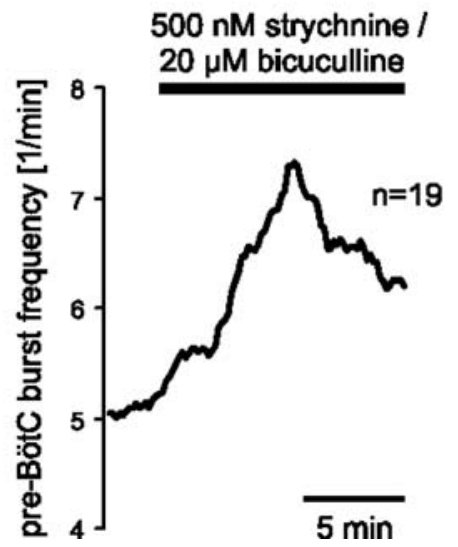

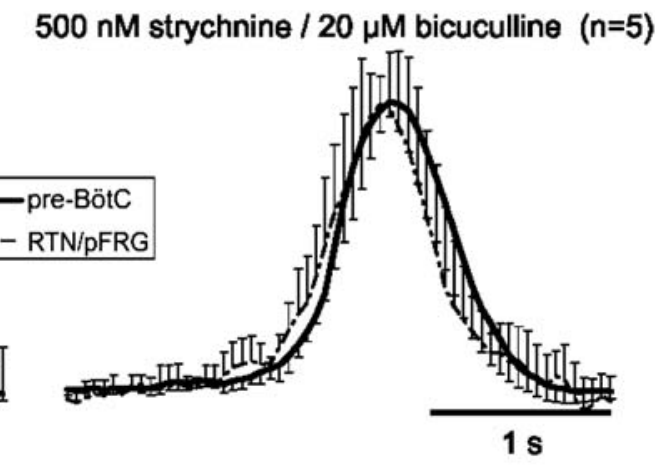

e

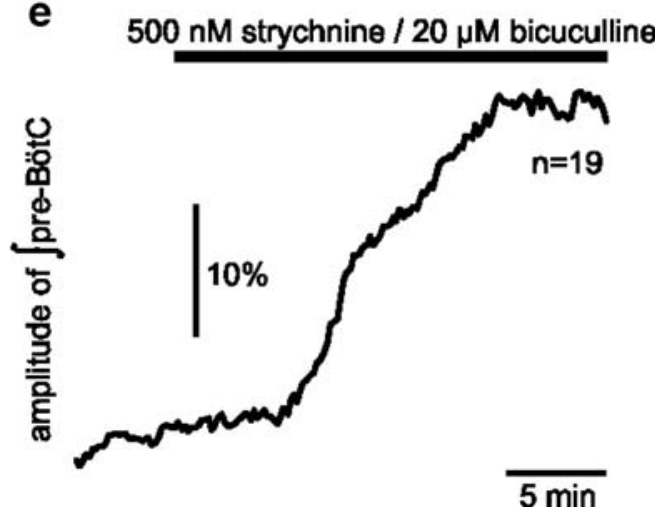


icant changes are marked by asterisks $(* P<0.05 ; * * P<0.01)$. Statistical calculations were done with the Excel software (Microsoft Office 2003).

\section{Results}

Anatomical description of the rostrally tilted transversal slice preparation

To verify the anatomical location of respiratory-related nuclei in the tilted slice preparation (cutting angle $=47^{\circ}$, $700 \mu \mathrm{m}$ thick), we cut $50-\mu \mathrm{m}$ sections using a freezing microtome $(n=5)$. Representative thionin-stained sections are illustrated in Fig. 1. For better orientation, please compare the thionin-stained sections (Fig. 1b-e) with their proposed location illustrated in the schematic drawing of a sagittal section through the pontomedullary brainstem (Fig. 1a). The first and the last two sections of the slice were not usable due to tissue damage. In the most caudal intact section, crucial landmarks for the pre-BötC in the ventral part of the slice preparation were the inferior olive (IO), the nucleus ambiguus (Amb), and the interpolar (ventral) and oral (dorsal) part of the spinal trigeminal nucleus (Sp5i). Due to the $47^{\circ}$ cutting angle, the nucleus ambiguus was visible at two different locations that represent the caudal (lateral) and more rostral (medial) aspects of this nucleus (see Fig. 1b). The nucleus ambiguus, together with the IO, allowed for the identification of the pre-BötC where the primary in vitro respiratory-like rhythm was recorded (see location of the recording electrode in the schematic drawing in Fig. 1b). In the next section, $100 \mu \mathrm{m}$ more rostral, the first caudalmost facial motoneurones could be identified (Fig. 1c). The schematic drawing of the recording electrode illustrates where we recorded rhythms of the RTN/pFRG. Please note that due to the cutting angle the facial motoneurones appear to be located slightly more dorsally and medially compared to conventional transversal slices (Fig. 1c). The next section, $100 \mu \mathrm{m}$ more rostral, already includes large pontine aspects of the respiratory network (Fig. 1d). This is illustrated by the presence of the inferior and superior colliculi which were easily identifiable. Furthermore, in these sections, the locus coeruleus (LC), the mesencephalic trigeminal neurones (Me5), and trigeminal motoneurones (Mo5) are visible and thus allow for the identification of the region of the $\mathrm{PB} / \mathrm{KF}$. The ventral parts of this section still include facial motoneurones. The last illustrated section (Fig. 1e) and most rostral sections are similar to the previous but LC, Mo5, and facial motoneurones begin to fade.
Recording of dual rhythms arising from the regions of the pre-BötC and RTN-pFRG

Simultaneous recordings of pre-BötC and RTN-pFRG mass activities ( $n=8$ slices) showed that their rising slopes differ significantly (see Fig. 2a) and that the RTN-pFRG burst onset preceded that of the pre-BötC in a time window of $350 \mathrm{~ms}$ (Fig. 2c).

Additionally, we recorded rhythmic mass activity from the region of the PB-KF. Rhythmic activity recorded from this region ranged from three bursts per minute to 20 bursts per minute. The bursts showed a decrementing pattern with highly variable length but showed no correlation with the pre-BötC rhythm. To further characterize a potential function of the KF in vitro, we electrically stimulated this region. In 3 out of 11 experiments, single pulse stimulation or stimulus trains which were applied in a time window of 100-500 ms before the expected pre-BötC burst suppressed a pre-BötC burst (data not shown). However, the majority of experiments did not show significant changes in preBötC rhythm. Therefore, we conclude that potential KFevoked resetting properties concerning the pre-BötC rhythm cannot be reliably studied in this slice preparation.

Synchronization of the dual rhythms following blockade of inhibitory neurotransmission

The effect of bath-applied strychnine $(500 \mathrm{nM})$ and bicuculline $(20 \mu \mathrm{M})$ on the activity of the oscillators located in the pre-BötC and RTN/pFRG was investigated in the present study and blockade of inhibitory neurotransmission revealed profound changes. The preburst component of the RTN-pFRG rhythm disappeared ( $n=5$ slices, Fig. 2c) due to a significant broadening of the pre-BötC bursts and the rising slopes of the pre-BötC and RTN/pFRG bursts synchronized (compare Fig. 2a with Fig. 2b). On average, the pre-BötC burst duration increased by $75 \pm$ $30 \mathrm{~ms}(n=5, p<0.05)$. Beside the synchronization of preBötC and RTN/pFRG rhythm, blockade of inhibitory neurotransmission caused a simultaneous increase in preBötC and RTN/pFRG burst frequency $(n=19)$. Initially, the mean frequency increased from $5.2 \pm 1.3$ to $7.0 \pm 2.7$ bursts per minute $(+35 \%, n=19, p<0.01$, Fig. 2 d). Ten minutes after application, the mean frequency stabilized at $6.3 \pm 1.9$ bursts per minute, still reflecting a significantly increased burst frequency compared to the baseline $(+12 \%, n=19, p<$ 0.01 , Fig. 2d). The mean amplitude of the integrated preBötC mass activity increased to $130 \pm 24 \%$ of the control amplitude ( $n=19, p<0.01$, Fig. 2e), stabilizing $15 \mathrm{~min}$ after strychnine-bicuculline application. The mean amplitude of 
Fig. 3 Classification of three different respiratory-related cell types. Rhythmic changes in $\left[\mathrm{Ca}^{2+}\right]_{\mathrm{i}}$ allowed for the identification of three different respiratory-related cell types. Each cell type is displayed by a frame sequence (1-5) showing the changes in pixel brightness superimposed onto a baseline image. The traces show the temporal changes in fluorescence intensity of the $\mathrm{Ca}^{2+}$ indicator Fluo-3 measured from the individual neurones. The grey box represents the mean duration of a pre-BötC burst. a Inspiratory-like neurones showed an increase in $\left[\mathrm{Ca}^{2+}\right]_{\mathrm{i}}$ in correlation with the pre-BötC burst (white arrowheads). $\mathbf{b}$ Neurones displaying a decrease in $\left[\mathrm{Ca}^{2+}\right]_{\mathrm{i}}$ during the pre-BötC burst were classified as expiratory-like (black arrowheads). c Preinspiratory neurones showed a rise in $\left[\mathrm{Ca}^{2+}\right]_{\mathrm{i}}$ clearly before the onset of the pre-BötC burst (white arrowhead) a
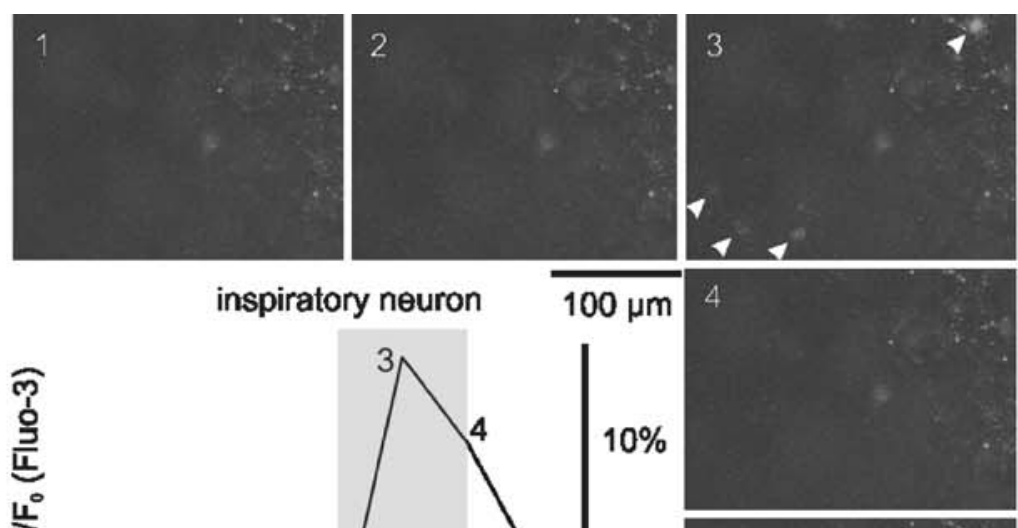

논

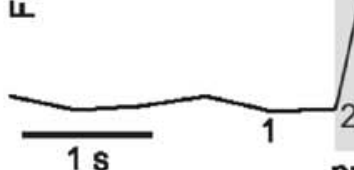

$1 \mathrm{~s}$

pre-BötC

burst

b
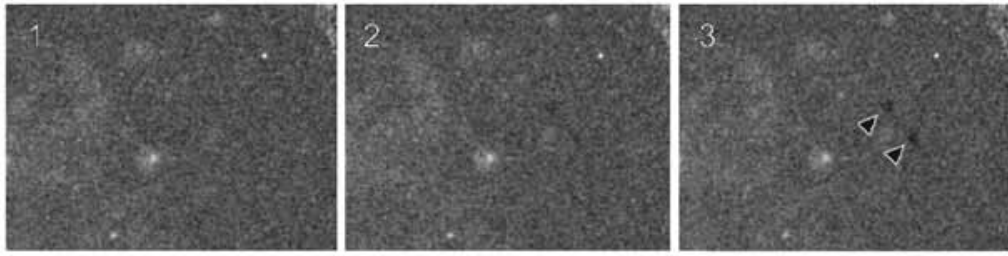

expiratory neuron

$100 \mu \mathrm{m}$
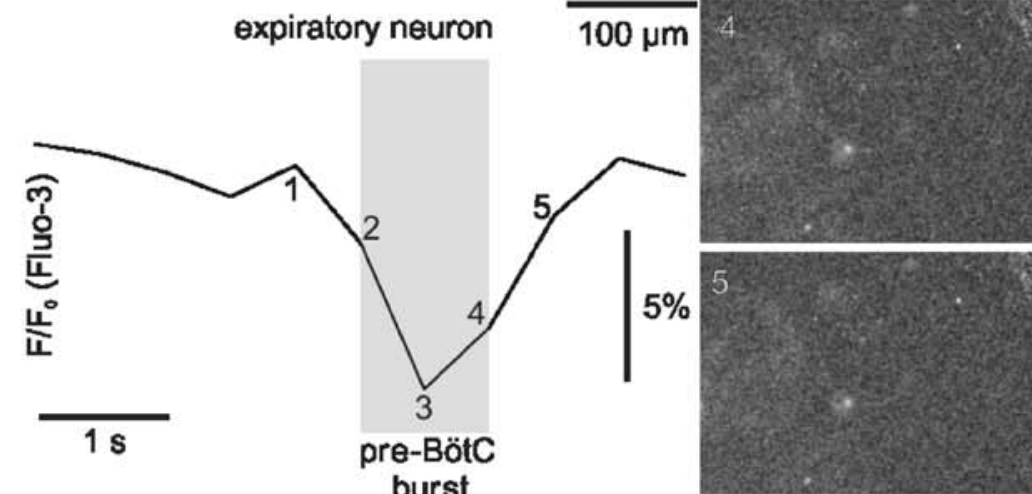

C

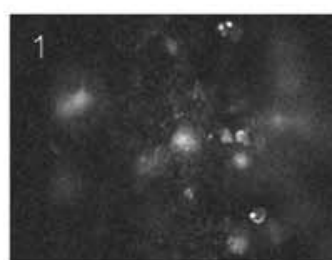

burst
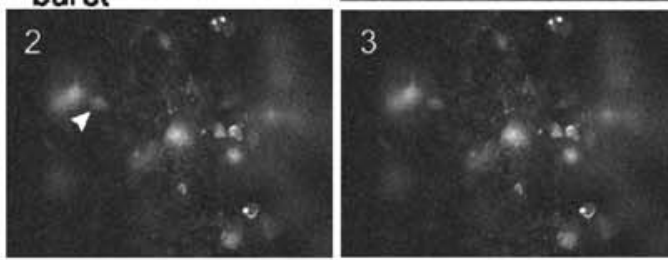

pre-inspiratory neuron

$100 \mu \mathrm{m}$
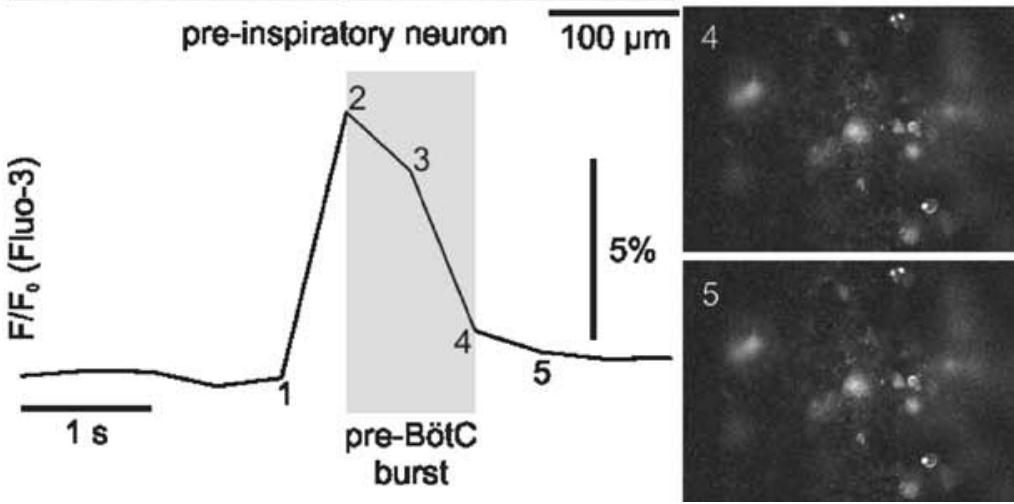
Fig. $4 \mathrm{Ca}^{2+}$ imaging revealed reconfigurations of cellular activity after blockade of inhibitory neurotransmission. a Inspiratory-like neurones that were not active during control recordings were recruited. This is illustrated by $\mathrm{Ca}^{2+}$ imaging of two neurones (arrowhead 1 and 2). During control activity, neurone 2 was not correlated with the pre-BötC burst (grey shadings in the diagrams in $\mathbf{a}$ and $\mathbf{b}$ ). After blockade of inhibitory neurotransmission by application of strychnine-bicuculline, the neurone became rhythmically active. The illustrated representative recordings were averaged over 28 consecutive bursts. Beside the recruitment, an increased $\mathrm{Ca}^{2+}$ rise in inspiratory neurones became obvious (compare the diagrams on the right side). b Expiratory-like neurones lost their pre-BötC mass activity correlated rhythmic inhibition after strychninebicuculline application (compare the diagrams on the right side). The displayed recordings are averaged over 22 consecutive pre-BötC bursts a

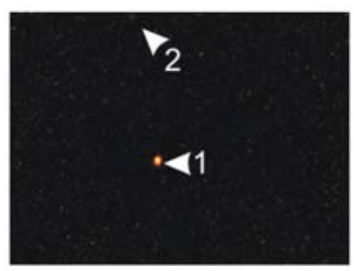

control

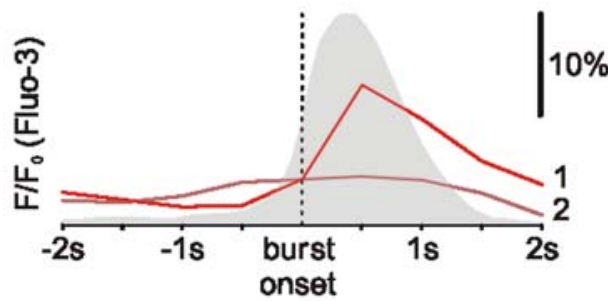

$500 \mathrm{nM}$ strychnine / $20 \mu \mathrm{M}$ bicuculline

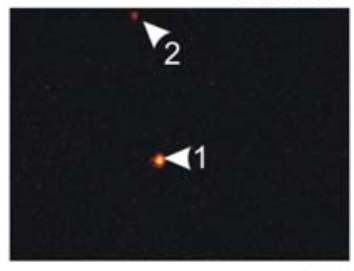

$5 \bar{\mu} \mathrm{m}$

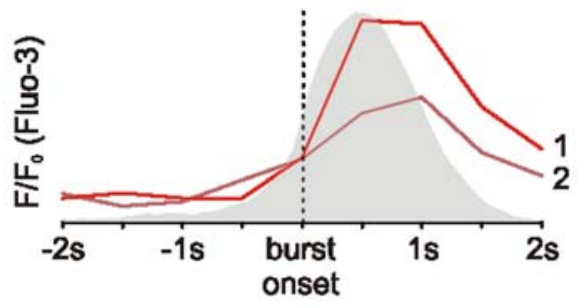

b

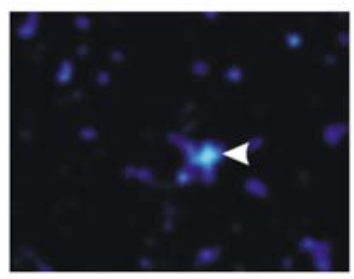

control

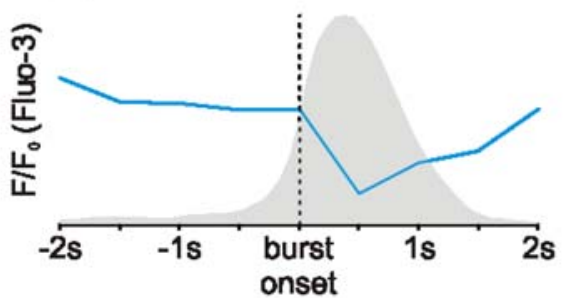

$500 \mathrm{nM}$ strychnine / $20 \mu \mathrm{M}$ bicuculline

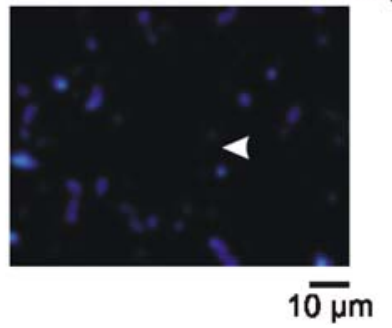

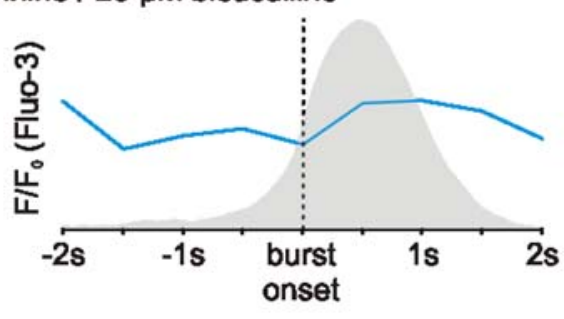

the integrated RTN/pFRG mass activity was unaffected $(n=8$, data not shown).

Reconfiguration of imaged cellular activity patterns following blockade of inhibitory neurotransmission

To identify changes in cellular activity underlying a potential reconfiguration of the respiratory-related rhythmic mass activity and its generation, we imaged population activity from the pre-BötC region. The analysis of changes in intracellular $\mathrm{Ca}^{2+}$ concentration $\left(\left[\mathrm{Ca}^{2+}\right]_{\mathrm{i}}\right)$ in relation to the recorded rhythmic pre-BötC mass activity in a total of $n=208$ imaged neurones allowed for the identification of three basic respiratory-related cell types. The vast majority of imaged neurones showed a rise in $\left[\mathrm{Ca}^{2+}\right]_{\mathrm{i}}$ during the preBötC burst $(n=159,76.4 \%)$ and were classified as inspiratory-like neurones (Fig. 3a). Neurones which showed a decrease in $\left[\mathrm{Ca}^{2+}\right]_{\mathrm{i}}$ during the pre-BötC burst were classified as expiratory-like neurones $(n=30,14.4 \%$, Fig. $3 b)$. Finally, we found a small fraction of neurones which showed their peak rise in $\left[\mathrm{Ca}^{2+}\right]_{\mathrm{i}}$ one imaged frame before the pre-BötC burst occurred $(n=19,9.2 \%)$. These neurones were classified as preinspiratory-like neurones (Fig. 3c).

According to the specific $\left[\mathrm{Ca}^{2+}\right]_{\mathrm{i}}$ fluctuations, different reconfigurations of cellular activities were observed after blockade of GABAergic and glycinergic neurotransmission. Recruitment of inspiratory-like neurones which were not active during the control recordings was found $(n=10$, neurone 2 in Fig. 4a). Inspiratory neurones which were already active during control showed a net increase in their $\left[\mathrm{Ca}^{2+}\right]_{i}$ rise (see neurone 1 in Fig. 4a). Both observed effects on inspiratory neurones contribute to the significant increase in burst amplitude of the rectified and integrated preBötC mass activity (see Fig. 2e). Furthermore, expiratory 
neurones lost their pre-BötC-associated decrease in $\left[\mathrm{Ca}^{2+}\right]_{\mathrm{i}}$ $(n=7$, Fig. $4 \mathrm{~b})$. The sampling rate of our imaging approach allows only for the detection of preinspiratory activity that occurred at least $500 \mathrm{~ms}$ before the inspiratory burst. Nevertheless, we occasionally observed neurones with this bursting behavior basically maintaining their activity pattern after strychnine and bicuculline application $(n=3$, data not shown), although their peak rise in $\left[\mathrm{Ca}^{2+}\right]_{\mathrm{i}}$ was in the early phases of the pre-BötC burst. The impression of a potential shift of their peak rise in $\left[\mathrm{Ca}^{2+}\right]_{\mathrm{i}}$ is misleading and actually caused by the significant broadening of the pre-BötC burst duration (see Fig. 2c).

We did not succeed to optically measure respiratoryrelated activity in the RTN/pFRG region. The neurones of interest in this area were located in deeper layers of the slice (see Fig. 1) and because the penetration depth of the used $\mathrm{Ca}^{2+}$ dye is limited to the upper $60 \mu \mathrm{m}$ of the tissue [34] they were most likely not stained. Thus, cellular activities from the RTN/pFRG were not imaged due to technical limitations.

\section{Discussion}

\section{Technical considerations}

The histological analysis of the rostrally tilted transversal slice preparation derived from neonatal rats, illustrates that the pre-BötC of the rostral ventrolateral medulla oblongata and the RTN/pFRG of the pontomedullary junction were preserved. Direct connections between these two rhythmogenic kernels remained functional due to their close proximity in the $700-\mu \mathrm{m}$-thick slice. The existence of two rhythms that synchronize after blockade of inhibitory neurotransmission further proves the integrity of the connectivity between the RTN/pFRG and pre-BötC. Nevertheless, it should be mentioned that indirect connections might be eliminated by the slicing procedure. A recent study [35] showed that acidosis influences the coupling states between the pre-BötC and the RTN/pFRG in the en bloc brainstem-spinal cord preparation of neonatal rats. Especially metabolic acidosis synchronized the peak activity of the RTN/pFRG to the inspiratory phase. Acidosis or hypoxia cause cessation of inhibitory neurotransmission [36-40] and, thus, mimics the effects that we observed after pharmacological blockade of inhibitory neurotransmission. The similarity of the results from the en bloc preparation and our rostrally tilted slice preparation provides further evidence that a functional connectivity between pre-BötC and RTN/pFRG was preserved in our study.

In addition to these two areas of the respiratory network the tilted transversal slice preparation contains parts of the pontine respiratory network, such as the $\mathrm{A} 5$ and the $\mathrm{PB} / \mathrm{KF}$ region. Rhythmic bursting in the $\mathrm{PB} / \mathrm{KF}$ region did, however, not correlate with the pre-BötC activity and thus was most likely not respiratory related. Nevertheless, phasic respiratory activity in the KF region was recorded in the en bloc spinal cord preparation [41] and transection of the dorsolateral pons triggered substantial changes in the respiratory pattern of neonatal rats $[42,43]$. Although the KF also shows clear signs of immaturity [44] during the early neonatal stages, the most plausible explanation for the lack of functionality of the KF in the tilted transversal slice preparation is that the fiber tracts which connect the KF with the RTN/pFRG and/or pre-BötC were not preserved. This assumption is further supported by our findings that electrical stimulation of the $\mathrm{PB} / \mathrm{KF}$ region failed to provoke a phase reset in the vast majority of experiments. The pontine $\mathrm{A} 5$ region may provide tonic inhibitory drive to the $\mathrm{RTN} / \mathrm{pFRG}$ and/or pre-BötC regions as it was described in the neonatal en bloc brainstem-spinal cord preparation [32]. This tonic drive could have contributed to the significantly lower burst frequency of the tilted transversal slice preparation ( $\sim 5$ bursts per minute) compared to previously published data on burst frequencies of the rhythmic pre-BötC slice preparation ( $\sim 8$ bursts per minute) under similar experimental conditions (e.g., $8 \mathrm{mM}$ extracellular $\left[\mathrm{K}^{+}\right], 27^{\circ} \mathrm{C}$ ).

It is in full accordance with data from the en bloc brainstem-spinal cord preparations that the mass activity recorded from the RTN/pFRG had a preinspiratory component which was not observed in the pre-BötC rhythm [27]. Preinspiratory neurones also displaying postinspiratory activity [45] were not observed. This can be explained by different experimental conditions (slice versus en bloc preparation), methodological aspects (mass activity recording versus imaging with a voltage-sensitive dye or intracellular recording) and age of the animals used. Nevertheless, the in vitro activities of the tilted transversal slice preparation introduced in this study may largely correspond to activity patterns recorded in the en bloc brainstem-spinal cord preparation. Finally, the imaging data demonstrate the presence of inspiratory-like, expiratory-like, and preinspiratory-like neurones in the slice as they were described in the en bloc preparation [32]. Only the expiratory activity in the en bloc preparation clearly shows more complexity [32, 46, 47], which is most likely due to the more conserved connectivity to the pontine respiratory circuits.

Configuration and reconfiguration of the in vitro network activity in the tilted transversal slice

Recent studies showed that the integrity of the pontomedullary respiratory network is required to generate the eupnoeic three-phasic motor pattern (inspiration, postinspiration, late expiration) of breathing [15, 23, 24]. Neverthe- 
less, specific compartments, which particularly include the pre-BötC, maintain their rhythmic activity in vitro [26], and such rhythmic slice preparations were extensively used to study cellular mechanisms of the primary respiratory-like rhythm generation. Previous studies showed that synaptic inhibition is functional in slices, but its contribution to the generation of the neonatal respiratory-related rhythm was considered to be marginal [21, 48, 49]. In that regard, our data are in full accordance with these previous studies because the in vitro rhythm never stopped after blockade of inhibitory neurotransmission. However, mass activities recorded from the pre-BötC and RTN-pFRG including optically recorded cellular population activities revealed that oscillatory mechanisms based on synaptic inhibition were partially preserved in the in vitro tilted transversal slice preparation.

The main findings of our study are in full accordance with previous reports that showed reconfigurations of in vitro network activity after blockade of inhibitory neurotransmission [36], including the loss of phasic inhibition in expiratory-like neurones [49] and recruitment of additional inspiratory-like pacemaker neurones [50]. In summary, the present data set supports the hypothesis that excitatory and inhibitory coupling between RTN/pFRG and pre-BötC [16, 30] is an essential mechanism for respiratory rhythm generation. Although it is discussed controversially [1719], the present data strengthen the hypothesis of Onimaru and Homma that the RTN/pFRG serves as the master generator of the in vitro respiratory-like rhythm because the pre-BötC activity is synchronized to that of the RTN-pFRG after blockade of synaptic inhibition, as it is implicated by the broadening of the pre-BötC burst. The RTN/pFRG has an important role for central chemoreception [51-53]. Thus, excitatory drive to the RTN/pFRG would fit to a chemoreceptor-induced increase of the inspiratory rate. Finally, recently published data from the perfused brainstem preparation of juvenile rats suggest that the interaction between the neuronal population of the RTN/pFRG and pre-BötC relate to the inspiratory ramp pattern generator $[3$, $15,23,24,54,55]$. This is also supported by the presence of excitatory drive from the preinspiratory-like to the inspiratory-like neuron populations of the pre-BötC, because the excitatory coupling of preinspiratory neurones to augmenting inspiratory neurones is indeed a hallmark for the initiation of the inspiratory ramp in hypothetical models of respiratory rhythm generation $\quad[3,23,24,54-56]$.

Acknowledgments We thank B. Hildebrandt for excellent technical assistance.

Grants This study was supported by grants of the DFGResearch Center Molecular Physiology of the Brain (M. Müller) and Berstein Center for Computational Neurosciences (01GQ0432, M. Dutschmann).
Open Access This article is distributed under the terms of the Creative Commons Attribution Noncommercial License which permits any noncommercial use, distribution, and reproduction in any medium, provided the original author(s) and source are credited.

\section{References}

1. McCrimmon DR, Ramirez JM, Alford S, Zuperku EJ (2000) Unraveling the mechanism for respiratory rhythm generation. Bioessays 22:6-9

2. Ramirez JM, Richter DW (1996) The neuronal mechanisms of respiratory rhythm generation. Curr Opin Neurobiol 6:817-825

3. Richter DW (1982) Generation and maintenance of the respiratory rhythm. J Exp Biol 100:93-107

4. von Euler C (1983) On the central pattern generator for the basic breathing rhythmicity. J Appl Physiol 55:1647-1659

5. Vasilakos K, Wilson RJ, Kimura N, Remmers JE (2005) Ancient gill and lung oscillators may generate the respiratory rhythm of frogs and rats. J Neurobiol 62:369-385

6. Fortin G, Charnay P, Champagnat J (2003) Linking respiratory rhythm generation to segmentation of the vertebrate hindbrain. Pflügers Arch 446:514-515

7. Ramirez JM, Viemari JC (2005) Determinants of inspiratory activity. Respir Physiol Neurobiol 147:145-157

8. Duffin J (2004) Functional organization of respiratory neurones: a brief review of current questions and speculations. Exp Physiol 89:517-529

9. Smith JC, Butera RJ, Koshiya N, Del Negro C, Wilson CG, Johnson SM (2000) Respiratory rhythm generation in neonatal and adult mammals: the hybrid pacemaker-network model. Respir Physiol 122:131-147

10. Richter DW, Spyer KM (2001) Studying rhythmogenesis of breathing: comparison of in vivo and in vitro models. Trends Neurosci 24:464-472

11. Feldman JL, Mitchell GS, Nattie EE (2003) Breathing: rhythmicity, plasticity, chemosensitivity. Annu Rev Neurosci 26:239-266

12. Ezure K (2004) Reflections on respiratory rhythm generation. Prog Brain Res 143:67-74

13. Feldman JL, Del Negro CA (2006) Looking for inspiration: new perspectives on respiratory rhythm. Nat Rev Neurosci 7:232-242

14. Bianchi AL, Denavit-Saubie M, Champagnat J (1995) Central control of breathing in mammals: neuronal circuitry, membrane properties, and neurotransmitters. Physiol Rev 75:1-45

15. Smith JC, Abdala AP, Koizumi H, Rybak IA, Paton JF (2007) Spatial and functional architecture of the mammalian brainstem respiratory network: a hierarchy of three oscillatory mechanisms. J Neurophysiol 98:3370-3387

16. Onimaru H, Ikeda K, Kawakami K (2007) Defective interaction between dual oscillators for respiratory rhythm generation in $\mathrm{Na}^{+}, \mathrm{K}^{+}$-ATPase $\alpha 2$ subunit-deficient mice. J Physiol 584:271284

17. Onimaru H, Homma I, Feldman JL, Janczewski WA (2006) Point: counterpoint: the parafacial respiratory group ( $\mathrm{pFRG}$ )/ pre-Bötzinger complex (preBötC) is the primary site of respiratory rhythm generation in the mammal. J Appl Physiol 100:2094-2095

18. Ramirez JM, Garcia A 3rd (2007) Point: medullary pacemaker neurons are essential for both eupnea and gasping in mammals. $\mathrm{J}$ Appl Physiol 103:717-718

19. Paton JF, St-John WM (2007) Counterpoint: medullary pacemaker neurons are essential for gasping, but not eupnea, in mammals. J Appl Physiol 103:718-720 
20. Paton JF, Ramirez JM, Richter DW (1994) Functionally intact in vitro preparation generating respiratory activity in neonatal and mature mammals. Pflügers Arch 428:250-260

21. Paton JF, Richter DW (1995) Role of fast inhibitory synaptic mechanisms in respiratory rhythm generation in the maturing mouse. J Physiol 484:505-521

22. Dutschmann M, Paton JF (2002) Glycinergic inhibition is essential for co-ordinating cranial and spinal respiratory motor outputs in the neonatal rat. J Physiol 543:643-653

23. Rybak IA, Shevtsova NA, Paton JF, Dick TE, St-John WM, Morschel M, Dutschmann M (2004) Modeling the pontomedullary respiratory network. Respir Physiol Neurobiol 143:307-319

24. Rybak IA, Abdala AP, Markin SN, Paton JF, Smith JC (2007) Spatial organization and state-dependent mechanisms for respiratory rhythm and pattern generation. Prog Brain Res 165:201-220

25. Koshiya N, Smith JC (1999) Neuronal pacemaker for breathing visualized in vitro. Nature 400:360-363

26. Smith JC, Ellenberger HH, Ballanyi K, Richter DW, Feldman JL (1991) Pre-Bötzinger complex: a brainstem region that may generate respiratory rhythm in mammals. Science 254: $726-729$

27. Onimaru H, Homma I (2003) A novel functional neuron group for respiratory rhythm generation in the ventral medulla. J Neurosci 23:1478-1486

28. Mellen NM, Janczewski WA, Bocchiaro CM, Feldman JL (2003) Opioid-induced quantal slowing reveals dual networks for respiratory rhythm generation. Neuron 37:821-826

29. Janczewski WA, Feldman JL (2006) Novel data supporting the two respiratory rhythm oscillator hypothesis. J Neurophysiol 96:1-2

30. Janczewski WA, Feldman JL (2006) Distinct rhythm generators for inspiration and expiration in the juvenile rat. J Physiol 570:407-420

31. Onimaru H, Arata A, Homma I (1988) Primary respiratory rhythm generator in the medulla of brainstem-spinal cord preparation from newborn rat. Brain Res 445:314-324

32. Ballanyi K, Onimaru H, Homma I (1999) Respiratory network function in the isolated brainstem-spinal cord of newborn rats. Prog Neurobiol 59:583-634

33. Barnes BJ, Tuong CM, Mellen NM (2007) Functional imaging reveals respiratory network activity during hypoxic and opioid challenge in the neonate rat tilted sagittal slab preparation. J Neurophysiol 97:2283-2292

34. Funke F, Dutschmann M, Müller M (2007) Imaging of respiratory-related population activity with single-cell resolution. Am J Physiol Cell Physiol 292:C508-C516

35. Okada Y, Masumiya H, Tamura Y, Oku Y (2007) Respiratory and metabolic acidosis differentially affect the respiratory neuronal network in the ventral medulla of neonatal rats. Eur J Neurosci 26:2834-2843

36. Lieske SP, Thoby-Brisson M, Telgkamp P, Ramirez JM (2000) Reconfiguration of the neural network controlling multiple breathing patterns: eupnea, sighs and gasps. Nat Neurosci 3:600-607

37. Ramirez JM, Quellmalz UJ, Wilken B, Richter DW (1998) The hypoxic response of neurones within the in vitro mammalian respiratory network. J Physiol 507:571-582

38. Schmidt C, Bellingham MC, Richter DW (1995) Adenosinergic modulation of respiratory neurones and hypoxic responses in the anaesthetized cat. J Physiol 483:769-781
39. England SJ, Melton JE, Douse MA, Duffin J (1995) Activity of respiratory neurons during hypoxia in the chemodenervated cat. $\mathrm{J}$ Appl Physiol 78:856-861

40. Schmid K, Bohmer G, Gebauer K (1991) Glycine receptormediated fast synaptic inhibition in the brainstem respiratory system. Respir Physiol 84:351-361

41. Kobayashi S, Onimaru H, Inoue M, Inoue T, Sasa R (2005) Localization and properties of respiratory neurons in the rostral pons of the newborn rat. Neuroscience 134:317-325

42. Fung ML, St John WM (1995) The functional expression of a pontine pneumotaxic centre in neonatal rats. J Physiol 489:579591

43. Dutschmann M, Wilson RJ, Paton JF (2000) Respiratory activity in neonatal rats. Auton Neurosci 84:19-29

44. Dutschmann M, Morschel M, Kron M, Herbert H (2004) Development of adaptive behaviour of the respiratory network: implications for the pontine Kölliker-Fuse nucleus. Respir Physiol Neurobiol 143:155-165

45. Onimaru H, Kumagawa Y, Homma I (2006) Respiration-related rhythmic activity in the rostral medulla of newborn rats. J Neurophysiol 96:55-61

46. Arata A, Onimaru H, Homma I (1998) Possible synaptic connections of expiratory neurons in the medulla of newborn rat in vitro. Neuroreport 9:743-746

47. Iizuka M (1999) Intercostal expiratory activity in an in vitro brainstem-spinal cord-rib preparation from the neonatal rat. $\mathrm{J}$ Physiol 520:293-302

48. Ramirez JM, Quellmalz UJ, Richter DW (1996) Postnatal changes in the mammalian respiratory network as revealed by the transverse brainstem slice of mice. J Physiol 491:799-812

49. Shao XM, Feldman JL (1997) Respiratory rhythm generation and synaptic inhibition of expiratory neurons in pre-Bötzinger complex: differential roles of glycinergic and GABAergic neural transmission. J Neurophysiol 77:1853-1860

50. Tryba AK, Pena F, Ramirez JM (2003) Stabilization of bursting in respiratory pacemaker neurons. J Neurosci 23:3538-3546

51. Guyenet PG, Mulkey DK, Stornetta RL, Bayliss DA (2005) Regulation of ventral surface chemoreceptors by the central respiratory pattern generator. J Neurosci 25:8938-8947

52. Li A, Nattie E (2002) CO2 dialysis in one chemoreceptor site, the RTN: stimulus intensity and sensitivity in the awake rat. Respir Physiol Neurobiol 133:11-22

53. Mulkey DK, Talley EM, Stornetta RL, Siegel AR, West GH, Chen X, Sen N, Mistry AM, Guyenet PG, Bayliss DA (2007) TASK channels determine $\mathrm{pH}$ sensitivity in select respiratory neurons but do not contribute to central respiratory chemosensitivity. J Neurosci 27:14049-14058

54. Richter DW, Ballanyi K, Schwarzacher S (1992) Mechanisms of respiratory rhythm generation. Curr Opin Neurobiol 2:788793

55. Bradley GW, von Euler C, Marttila I, Roos B (1975) A model of the central and reflex inhibition of inspiration in the cat. Biol Cybern 19:105-116

56. Ezure K (1990) Synaptic connections between medullary respiratory neurons and considerations on the genesis of respiratory rhythm. Prog Neurobiol 35:429-450

57. Kron M, Morschel M, Reuter J, Zhang W, Dutschmann M (2007) Developmental changes in brain-derived neurotrophic factormediated modulations of synaptic activities in the pontine Kölliker-Fuse nucleus of the rat. J Physiol 583:315-327 